\title{
Zum Inhalt von Band V
}

Im vorliegenden fünften Band „Quanten, Atome, Kerne, Teilchen“ geht es um die moderne Physik. Im atomaren und subatomaren Bereich sind die Größen und Vorgänge nicht mehr kontinuierlich, sondern haben einen quantenhaften Charakter. Dies zeigen der Photo- und der Compton-Effekt für das elektromagnetische Strahlungsfeld, das in diesen Fällen als „körnig“, aus Photonen, kleinsten Portionen des elektromagnetischen Strahlungsfeldes bestehend, gedacht werden muss. Umgekehrt muss zur Erklärung der „Selbstinterferenz“ von Materieteilchen angenommen werden, dass diese mit - immateriellen - „Materiewellen“ verknüpft sind. Bei geeigneter Formulierung gibt das Absolutquadrat der Wellenfunktion dieser Materiewellen Auskunft über den Aufenthaltsort des Teilchens. Diese Vorstellung ermöglicht auch ein Verständnis der Vorgänge der stimulierten Emission beim Laser. Die Atomspektren und die Stoßexperimente von Franck und Hertz zeigen, dass auch beim Modell eines Atoms klassische Vorstellungen abgelegt werden müssen. Die Schrödingergleichung ist die Wellengleichung für die i. Allg. komplexen Materiewellen. Damit können der Aufbau und die Eigenschaften der Atome verstanden werden. Dies wird am Beispiel des Wasserstoffatoms gezeigt. Abschließend wird der Atomkern genauer betrachtet und die verschiedenen Modellvorstellungen dazu werden erläutert. Radioaktiver Zerfall, Kernspaltung und Kernfusion werden besprochen und ihre Anwendungen diskutiert. Schließlich werden die kleinsten, aus heutiger Sicht nicht mehr weiter teilbaren „Fundamentalteilchen“, aus denen sich alle Arten von Materie und Antimaterie zusammensetzen, vorgestellt und ihre Eigenschaften und Wechselwirkungen studiert. Der Band schließt mit einem kurzen Ausflug in die Kosmologie und zeigt die derzeitigen Vorstellungen über die Entwicklung unseres Universums. 
